\title{
Maximizing Energy Efficiency for Consumption Circuit Power in Downlink Massive MIMO Wireless Networks
}

\author{
Adeeb Salh, Lukman Audah, Nor Shahida M. Shah, Shipun A. Hamzah \\ Wireless and Radio Science Centre (WARAS), Faculty of Electrical and Electronic Engineering, \\ Universiti Tun Hussein Onn Malaysia, 86400 Parit Raja, Batu Pahat, Johor, Malaysia
}

\begin{tabular}{l} 
Article Info \\
\hline Article history: \\
Received Jun 8, 2017 \\
Revised Aug 30, 2017 \\
Accepted Sep 14, 2017 \\
\hline
\end{tabular}

Keyword:

$5 \mathrm{G}$ cellular systems

Enrgy efficiency

Multi-input

Multi-output

Quality of service

Signal-to-noise ratio

\begin{abstract}
Massive multi-input-multi-output (MIMO) systems are crucial to maximizing energy efficiency (EE) and battery-saving technology. Achieving EE without sacrificing the quality of service (QoS) is increasingly important for mobile devices. We first derive the data rate through zero forcing (ZF) and three linear precodings: maximum ratio transmission (MRT), zero forcing (ZF), and minimum mean square error (MMSE). Performance EE can be achieved when all available antennas are used and when taking account of the consumption circuit power ignored because of high transmit power. The aim of this work is to demonstrate how to obtain maximum EE while minimizing power consumed, which achieves a high data rate by deriving the optimal number of antennas in the downlink massive MIMO system. This system includes not only the transmitted power but also the fundamental operation circuit power at the transmitter signal. Maximized EE depends on the optimal number of antennas and determines the number of active users that should be scheduled in each cell. We conclude that the linear precoding technique MMSE achieves the maximum EE more than ZF and MRT because the MMSE is able to make the massive MIMO system less sensitive to SNR at an increased number of antennas.
\end{abstract}

Copyright (C) 2017 Institute of Advanced Engineering and Science. All rights reserved.

\section{Corresponding Author:}

Lukman Audah,

Wireless and Radio Science Centre (WARAS),

Faculty of Electrical and Electronic Engineering,

Universiti Tun Hussein Onn Malaysia,

Parit Raja, 86400 Batu Pahat, Johor, Malaysia.

Email: hanif@uthm.edu.my

\section{INTRODUCTION}

The requirements of wireless communication cellular networks providing high data rates have increased proportionally with the introduction of smart mobile phones. EE is critical when taking account of circuit power consumption. EE in a multiple cellular system increases if the number of antennas adopted at the transmitter signal increases to infinity. A recent study shows around $80 \%$ of energy consumed in the cellular network at the base station (BS) requires redesigning the BS technology to decrease consumption power, which extends the working life of the battery in the terminal. EE is an important design quality for fifth-generation (5G) cellular systems [1], providing every user $K$ with high data rates in cellular networks without sacrificing the QoS. In 5G cellular networks, providing high throughput as well as EE is very important. Moreover, power consumption increases not only because of the transmitted power on the power amplifier but also according to more power consumption because of analog devices such as oscillators, mixers, and power amplifiers. To reduce hardware constraints created because of phase noise, noise amplification, and signal gain, these operators increase the cost of antennas in the BS, requiring the deployment of a large antenna array. This may reduce the cost of a conventional transmitter via hardware constraints [2-4]. The total power consumed greatly depends on the number of active antennas that should be 
deployed in the BS and the number of served users [3]. Here, we investigate how to minimize power consumption while achieving a high data rate by obtaining the optimum number of antennas. The transmitted power consumption, the size of hardware, and the effect of circuit power consumption because of radio frequency chain (RF) can all be decreased by using more antennas at the BS, which are able to use power amplifiers as a constraint to prevent drawbacks such as a nonideal amplifier using a massive MIMO system [5], [6].

In In a multicellular massive MIMO system, downlink transmission linear precoding techniques such as maximum ratio transmission beamforming (MRTBF), zero forcing beamforming (ZFBF), and minimum mean square error beamforming (MMSEBF) are able to mitigate interference and noise because of the expansion of the power received at low power transmission rates. The number of elements in the antenna (without affecting transmitted power consumption) can be decreased proportional to $1 / M$ if the BS has perfect channel state information (CSI) and by $1 / \sqrt{M}$ if the BS has imperfect CSI, with only a slight loss in data rates [7], [8]. Massive MIMO systems can provide very high throughput in a new generation of cellular networks.

EE can be optimal when the number of antennas $M \rightarrow \infty$ without accounting for consumption circuit power [9], [10]. The radiated power constraint can achieve a high data rate using power allocation [11].

\section{SYSTEM MODEL}

Downlink cellular massive MIMO systems have many cells $C=[1,2, \ldots, c]$, where the BS contains $c \in C$ and every cell uses $M$ antennas that serve many active users $K$. Every BS sends different data symbols to every active user $K$. Consequently, the joint transmission is incoherent, which becomes less complicated than coherent joint transmission.

The received signal for users can be expressed as follows,

$$
\gamma_{k}=\sum_{i=1}^{L} \sqrt{Q_{i, t}^{d l}} w_{i, k} z_{i, k}
$$

where $Q_{i, t}^{d l}$ is the downlink transmit power for every user.

The downlink transmission each active users (UEs) receives from all BS, which contains the desired signal, interference and noise can written as

$$
\gamma_{k}=\underbrace{\sum_{i=1}^{L} \sqrt{Q_{i, t}^{d l}} h_{i, k}^{H} w_{i, k} Z_{i, k}}_{\text {desired signal }}+\underbrace{\sum_{i=1}^{L} \sum_{t=1, t \neq k}^{K} \sqrt{Q_{i, t}} h_{i, k} w_{i, t} Z_{i, t}}_{\text {interference }} \underbrace{+N_{k}}_{\text {noise }}
$$

Here, the linear precoding vector $w_{l, t} \in C^{M}$ represents the signal transmitted to every UE, $Z_{l, t}$ represents the payload symbol user inside the location cell, the unit transmit power $\mathbb{E}\left\|Z_{l, t}\right\|^{2}=1$, and $Q_{m}=\operatorname{diag}\left[q_{1}, q_{2}, \ldots \ldots \ldots, q_{M}\right]$ is the transmitted power from the BS to every UE $K$ using power allocation. Where the received signal $Z_{k}=\left[z_{1}, z_{2}, \ldots \ldots \ldots, z_{K}\right]$, the BS uses the channel to estimate and precode the signal; let $H \in C^{K \times M}$ be the linear precoding channel matrix between the BS and UE $K h_{k}=\left[H_{k, 1}, H_{k, 2}, \ldots, H_{k, m}\right]$, and $N_{k}$ is additive white Gaussian noise.

The transmit power for every different users at transmitted payload symbol from BS to users is

$$
Q_{z}=\sqrt{Q_{i, t}^{d l}} h_{i, k}^{H} w_{i, k}
$$

To reduce the interference and noise in the transmitted signal to many users inside the cell, the transmit power in term interference can be expressed as

$$
Q_{I}=\sum_{i=1}^{L} \sum_{t=1, t \neq k}^{K} \sqrt{Q_{i, t}} \cdot h_{i, k} w_{i, t} Z_{i, t}+1
$$

The massive MIMO system sends different amounts of power to every active user, taking into account that power should be allocated [13]. The receipt of signal-to-interference-noise ratio (SINR) in terms of UEs is 
$\Gamma_{i, K}=\frac{\sqrt{Q_{i, t}^{d l}} \cdot h_{i, k}^{H} \cdot w_{i, k}}{\sum_{i=1}^{L} \sum_{t=1, t \neq k}^{K} \sqrt{Q_{i, t}} \cdot h_{i, k} \cdot w_{i, t} z_{i, t}+1}$

The achievable data rate for user can expressed as

$$
R_{k}\left(h_{k}\right)=B\left(1+\Gamma_{i, K}\right)
$$

where $B$ is the coherence bandwidth. follows:

To mitigate noise and interference, we use the linear precoding technique with prefect CSI [7] as

$$
\begin{aligned}
& M R T=h \\
& Z F=h\left(h^{H} h\right)^{-1} \\
& M M S E=h\left(h^{H} h+M I_{B}\right)^{-1}
\end{aligned}
$$

In downlink transmission, every user estimates the correlated received signal, and the SINR of users corresponds to the received signal for ZFBF [11]. This is because the ZF has low complexity and its performance is very close to that of maximum likelihood. In addition, good performance at high and low signal-to-noise ratios (SNR) in terms of data rate multiuser precoding is given as

$$
\Gamma_{i, K}=\frac{Q_{i, t}^{d l}}{\operatorname{tr}\left(\left(H^{H} H\right)^{-1}\right)}
$$

by

Based on the random matrix theory [12], the BS equipped with a massive MIMO system, is given

$$
\frac{1}{\operatorname{tr}\left(\left(H^{H} H\right)^{-1}\right)}=\frac{(M-K+1)}{K}
$$

The linear precoding zero forces beamforming to use perfect CSI, which achieves a data rate at the receiver when $M \geq K+1$, the data rate per user $K$ [14] as $r_{k}$ for all users is expressed as $R=\left[r_{1}, r_{2}, \ldots \ldots ., r_{k}\right]$, and Gaussian noise is 1 . The linear precoding technique with perfect CSI at active users adopts $\mathrm{ZF}$, which cancels the intracell interference; let us denote that the achievable data rate according to [15], [16] is given by

$$
R_{k}\left(h_{k}\right)=\sum_{k=1}^{K} r_{k}=\frac{B}{2}\left(1-\frac{2 K}{T}\right) \log _{2}\left[1+\frac{Q_{k}(M-K+1)}{K}\right]
$$

\subsection{Energy Efficiency}

In this section, we obtain maximum EE under minimum power consumption while achieving a high data rate by deriving the optimal number of antennas in the downlink multiple cellular massive MIMO system.

Problem: To obtain maximum EE by minimizing power consumption but achieving a high throughput to total power consumption, energy efficiency can be expressed as

$$
E E^{o p t}=\arg \max _{Q} E E(Q)=\arg \max _{Q} \frac{\max _{Q} R\left(h_{k}\right)}{Q_{C P}^{t o t}(K, M)}
$$

The average data rate per user when $K$ active users are present in the cell while checking the channel estimate is $h_{k}$. Maximum EE requires knowing how many antennas must be employed, depending on the number of active users scheduled in each cell. To obtain maximum EE, one needs to find the optimal number of antennas when the number of active users is fixed at $M, K$ to $(0, \infty)$

$$
\begin{gathered}
\max _{E E^{o p t} \geq 0, Q \in \max _{Q_{k}} R\left(h_{k}, \gamma_{k}\right)} E E(Q) \\
\text { s.t. }\left(E E^{\max }, Q_{C P}^{\text {tot }}(K, M)\right) \geq 0, \\
K \leq M .
\end{gathered}
$$




\subsection{Minimize Consumption Power}

The total power consumption in RF because of the long haul of transmitted data and local receiver side can be divided into three main components, viz. power consumption for all power amplifiers $Q_{P A}(Q)$, the base band signal processing $Q_{B B}$ when BS provides many $M$ s to serve $K$ simultaneously, and $Q_{C P}(Q)$ consumption in all circuit blocks. Thus, the total consumption power is given by

$$
\begin{gathered}
\min _{M_{k, o p t}} Q_{C P}^{t o t}(K, M)=M_{N} Q_{P A}(Q)+Q_{B B}(K, M)+Q_{C P}(Q) \leq \frac{Q_{C P}^{t o t}(K, M)}{M_{k, o p t}} \\
\text { s.t. } Q_{C P}^{t o t}(K, M) \geq 0 \\
Q_{C P}=Q_{L P}+Q_{f i x}+Q_{C E}+Q_{C / D}+Q_{R F}
\end{gathered}
$$

According to the previos work in [3], from (15), where $Q_{L P}$ is the linear processing in the BS, $Q_{f i x}$ is a constant (a fixed consumption power). $Q_{C E}$ denotes the channel estimation, which evaluates and computes processing efficiency between the BS and active users $K ; Q_{C / D}$ is the decoding and encoding channel.

The consumption power in the RF chains $Q_{R F}$ due to noise amplification is

$$
Q_{R F}=M Q_{B S}+2 K Q_{U E}+Q_{s y n}
$$

From (16), the power consumption at the RF is affected by the power consumption at the BS $\left(Q_{B S}\right)$, $\operatorname{UE}\left(Q_{U E}\right)$, and the local synchronous oscillators $\left(Q_{s y n}\right)$.

To evaluate the power consumption of RF chains using beamforming at every antenna for each active user $K$, the effective circuit power in the BS is given by

$$
Q_{B S}=Q_{0}+M Q_{S}+\Delta_{Q} Q_{t}
$$

where $Q_{0}$ is the consumption power at $\mathrm{BS}, Q_{S}$ is the consumption power at every antenna at the $\mathrm{BS}, \Delta_{Q}$ is the coefficient transmitted power, and $Q_{t}$ the total transmitted power from BS to every active user $K$. The transmitted power and circuit power consumption during the downlink for one user is given by

$$
Q_{U E}=\frac{B}{S} \sum_{k=1}^{K} \frac{Q_{C U}}{\eta_{U}}
$$

where $Q_{C U}$ is the power consumed in the circuit components for each user, $\eta_{U}=\eta Q_{P A}$ represents effective power amplifier efficiency, and $S$ is the coherence length. The local synchronous oscillators are given by

$$
Q_{\text {syn }}=\delta_{0,0}=Q_{f i x}
$$

where, $\delta_{0,0}$ represents constant coefficient circuit power.

Based on (14), the power consumption by the power amplifier is expressed as

$$
Q_{P A}(Q)=\frac{1}{\eta}\left(Q_{r} \cdot Q_{\max , P A}\right)^{1 / 2}
$$

where $\eta$ represents the maximum power amplifier efficiency, when transmitting the maximum output power and $Q_{r}$ represents the average power,

$$
\eta=\frac{1}{\left(1-\tau_{c o o l}\right)\left(1-\tau_{d c}\right)\left(1-\tau_{m s}\right)}
$$

where, $\eta$ represents the independent power for cooling, main power supply, and DC-DC converter loss.

From (14), the total power consumed in terms of base band processing power $Q_{B B}$ because of the conversion from ADC/DAC can expressed as

$$
Q_{B B}(M, K)=\frac{Q_{i}}{\eta_{i}} \partial K+\sum_{m=0}^{3} \delta_{0, m} K^{m}+M \sum_{i=0}^{2} \mathrm{~W}_{1, m} K^{m}, \quad i=1
$$

where, $\eta_{i}$ represents the efficiency of the power amplifier of BS, while $\partial$ represents the power consumption in terms of both decoding and encoding, 


$$
\partial=Q_{C O D}+Q_{D E C}+Q_{B T}
$$

where $Q_{B T}$ is the power required for backhauling traffic and $\partial$ is again the power consumption in terms of both decoding and encoding.

Maximum energy efficiency is given in terms of zero forcing beamforming precoding, which minimizes the received interference power.

In this section, we investigate how to maximize EE while minimizing power consumption by deriving the optimal number of antennas in the downlink multiple cellular massive MIMO system. Maximized EE depends on the optimal number of antennas and determines the number of active users that should be scheduled in each cell.

Theorem 1. Maximum EE requires knowing that the optimal number of antennas $M$ must be served; it depends on the number of active users $K$ and should be scheduled in each cell $M^{\text {opt }}=$ Round $\left(\max \left(M^{*}, K\right)\right)$.

$$
\frac{\partial E E\left[\max _{Q_{k}} R\right]}{\partial M}\left(M_{N} Q_{P A}(Q)+Q_{B B}(K, M)+Q_{C P}\right)-\max _{Q_{k}} R\left[Q_{C P}\right]=0
$$

Using a high degree of freedom while ignoring the constraint $K \leq M$, we can use (11) by using its second derivative, given in (24), in addition to minimizing the power consumed.

$$
\frac{\partial^{2} E E\left[\max _{Q_{k}} R\right]}{\partial M^{2}}\left(M_{N} Q_{P A}(Q)+Q_{B B}(K, M)+Q_{C P}\right)
$$

By using a high degree of freedom, the interval $(K-1, \infty)$ can be substituted in terms of the number of antennas $M$, where $[M=K-1, \infty]$. From the second derivative, the function $\left.\frac{\partial^{2} E E\left[\max _{Q_{k}} R\right]}{\partial M^{2}}\right|_{M=K-1}$ decreases for $M$ when $M \rightarrow K-1$, and becomes

$$
\left.\frac{\partial^{2} E E\left[\max _{Q_{k} R}\right]}{\partial M^{2}}\right]_{M=K-1}\left(M_{N} Q_{P A}(Q)+Q_{B B}(K, M)+Q_{C P}\right)>0
$$

If the number of antennas $M \rightarrow \infty$, the first derivative is $\lim _{M \rightarrow \infty} \frac{\partial E E\left[\max _{Q_{k}} R\right]}{\partial M} 1_{M \rightarrow \infty}=0$ and the second derivative of the first term of (14) is

$$
\lim _{M \rightarrow \infty} \frac{\partial^{2} E E\left[\max _{Q_{k}} R\right]}{\partial M^{2}}=0-\operatorname{limmax}_{M \rightarrow \infty} R\left[Q_{Q_{k}}\right]<0
$$
downlink

To analyze, the maximized EE is based on power consumption and a higher data rate in the

$$
E E_{M \geq K}^{\operatorname{maximize}}=\frac{\sum_{k=1}^{K} \log _{2}\left(1+\frac{Q_{k}(M-K+1)}{K}\right)}{M_{N} Q_{P A}(Q)+Q_{B B}(K, M)+Q_{C P}^{Z F}}
$$

$$
\text { Subject to } K+1 \leq M
$$

From (28), in the denominator, we analyze power consumption in the third term $Q_{C P}^{Z F, M R T, M M S E}$ using transmitter beamforming techniques, which can be analyzed using three types-MRTBF, ZFBF, and MMSEBF - allowing us to reduce the signal energy for the transmitted signals [17]. To analyze the transmitted power consumption based on linear processing beamforming, the beamforming can be applied in a non-line of sight $(\mathrm{LoS})$ between the transmitter and the receiver. In addition, if the LoS in the multipath channel is known, $\mathrm{Q}_{\mathrm{LP}}^{\mathrm{ZFF}}$ at the $\mathrm{BS}$ using linear precoding zero forcing beamforming can be expressed as 


$$
Q_{C P}^{Z F B F}=Q_{B B}^{Z F B F}+Q_{L P}^{Z F B F}
$$

The maximum EE is inversely proportional to the consumed transmitted power and proportional to the number of antennas $M$ and UEs $K$. Therefore, to reduce the power consumed, we can use the following equation to avoid the effect of consumed power,

$$
Q_{B B}^{Z f B F}=\sum_{m=0}^{3} \delta_{m} k^{m}+M \sum_{I=0}^{2} \mathrm{~W}_{m} k^{m}+K \partial \frac{Q_{i}}{\eta_{i}}
$$

where $\delta_{i}$ and $W_{i}$ represent constant coefficient circuits. The maximum EE is improved by increasing the number of antenna elements in the BS according to the convex optimization theory using the linear processing beamforming power consumption $Q_{L P}{ }^{M R T B F}, Q_{L P}{ }^{Z F B F}$, and $Q_{L P}{ }^{M M S E B F}$ [18-20]. The BS applies a linear receiving combination to discriminate the signal transmitted by each terminal from the interfering signals and the transmitter that is generated at the BS using a linear transmitter, which maximizes the EE [19].

Proposition 1. For $Q_{L P}{ }^{M R T B F}, Q_{L P}{ }^{Z F B F}$, and $Q_{L P}{ }^{M M S E B F}$, the transmitted linear processing power consumption with $M$ and $K$ is

$$
Q_{l p}^{M R T B F, Z F B F, M R T B F}\left\{\begin{array}{l}
B\left(1-\frac{\beta K}{S}\right) \frac{2 M k}{L_{B S}}+\frac{\beta K}{S} \frac{3 M K}{L_{B S}} \text { for } M R T B F \\
\frac{B}{S}\left(\frac{\beta}{T} \frac{8 M k^{3}}{3 * L_{B S}}+B \frac{3 M K^{2}}{L_{B S}}-B \frac{6}{T} \frac{M K^{5}}{L_{B S}}\right) \text { for } Z F B F \\
B\left(1-\frac{\beta K}{S}\right) \frac{2 M k}{L_{B S}}+\frac{\beta K}{S} \frac{3 M K}{L_{B S}} \text { for } M M S E B F
\end{array}\right.
$$

where $T$ is the channel coherence time in s, $B$ is the coherence bandwidth, $\beta$ is the pilot sequence transmitted, and $S$ is the coherence interval given as $S=B_{C} T_{C}$.

The linear processing in the BS has to search for all possible transmitted signal vectors and chooses the best one depending on the number of antennas $M$ of the BS and on the number of active users who should be scheduled in each cell $K$ inside $L_{B S}$, according to (31), where $Q_{L P}$ is inversely proportional to the same local cell $L_{B S}$.

From Proposition 1, the power consumption during linear processing beamforming is affected by both the number of antennas $M$ and the number of active users $K$. The maximized energy efficiency can be expressed as

$$
E E_{M \geq K}^{Z F B F}=\frac{\boldsymbol{K}\left(\mathbf{1}-\frac{\beta K}{S}\right) \sum_{k=1}^{K} \log _{2}\left(1+\frac{Q_{k}(M-K+1)}{K}\right)}{\underbrace{\sum_{m=0}^{3} \delta_{m} k^{m}+M \sum_{m=0}^{2} W_{m} k^{m}+K \partial \frac{Q_{i}}{\eta_{i}}+\underbrace{\frac{1}{\eta}\left(Q \cdot Q_{m a x, P A}\right)^{1 / 2}}_{Q_{P A}(Q)}+\underbrace{\left.\frac{\beta}{S}\left(\frac{B 8 M k^{3}}{T 3 * L_{B S}}+B \frac{3 M K^{2}}{L_{B S}}-B \frac{6 M K^{5}}{T L_{B S}}\right)\right)}_{Q_{l P} Z \mathbf{B F}}}_{Q_{B B}^{Z f B F}}}
$$

\section{NUMERICAL RESULT}

From Figure 1, when increasing the number of users and antennas with the same transmitted power, the array gain increases linearly. The improved array gain can be increased under massive MIMO system, while the line of sight after increasing the number of antennas to $100 \mathrm{M}$ is still saturated. The increment of array gain will improve the QoS and coverage area. Consequently, the number of antenna elements in the case of the line of sight increases, and the transmitted signal also increases based on the state of the channel model and available optimal transmitted power. Meanwhile, we take account of the array gain; in massive MIMO systems, it provides better orthogonality between channels for the transmitted signal to many users $K$. In addition, the array gain has increased the coverage area and reduced the consumption curcit power.

From Figure 2, the achievable data rate with perfect CSI changes by increasing the number of antennas $M$, showing that the number of active users should be scheduled in each cell because of the transmitted power. The linear precoding MMSE gives a high data rate, more than ZF and MRT. We can state that MMSE and ZF are able to work at high SINR when the number of antenna arrays increases, because adding more antennas will only enhance the SINR. From Figure 2, when the number of $M=140$, the achievable data rate starts showing the same value depending on the scheduled number of usres and number of antennas in each cell. 
From Figure 3, maximum EE clearly depends on choosing the optimal number of antennas for use with linear precoding techniques, such as MMSE, which obtain maximum EE at an optimal number of antennas $M^{o p t}=29$. In contrast, for ZF techniques, maximum EE can be obtained for a number of optimal antennas $M^{o p t}=33$ and MRT at $M^{o p t}=38$. From Figure 3, after obtaining high EE, efficiency will begin to decrease because of the increasing number of antennas at the BS. The MMSE gives better performance because the MMSE is able to make the massive MIMO system less sensitive to SNR at an increased number of antennas for achievable data rates compared with ZF and MRT, where an MMSE and ZF are able to work at high SNR. Consequently, EE first increases and then decreases with an increased number of antennas, which maximizes the transmit power $Q_{t}$ when taking into account the consumption circuit power and the transmit power. In addition, increasing the number of antennas inside the cell will increase the transmit power and operating power consumption, which creates the tendency of a concave shape for EE.

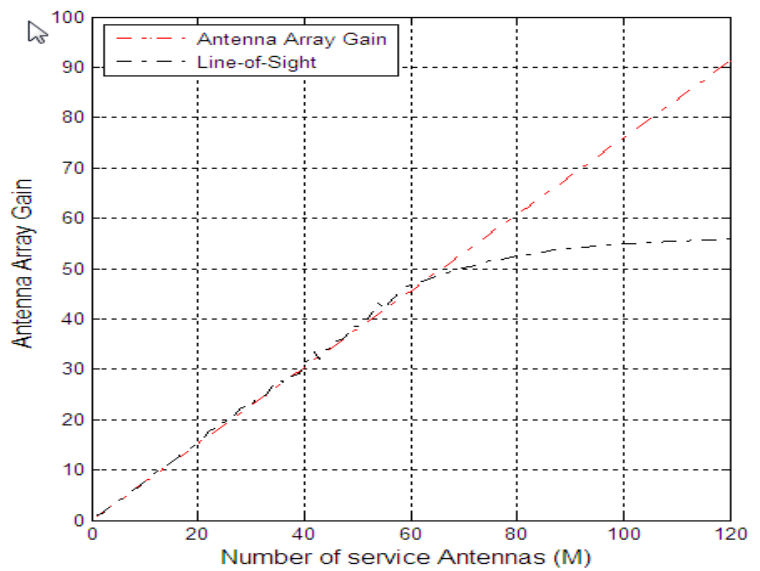

Figure 1. Antenna array gain with number of service antennae $M$

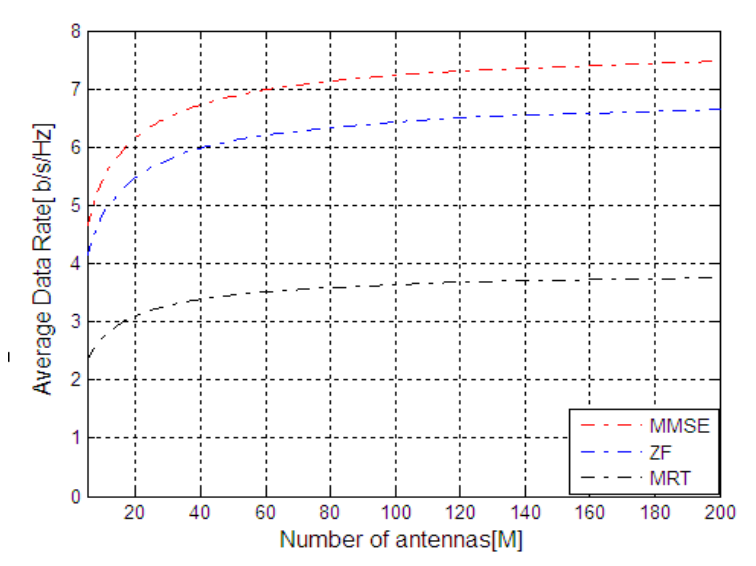

Figure 2. Average data rate with number of antennas $M$

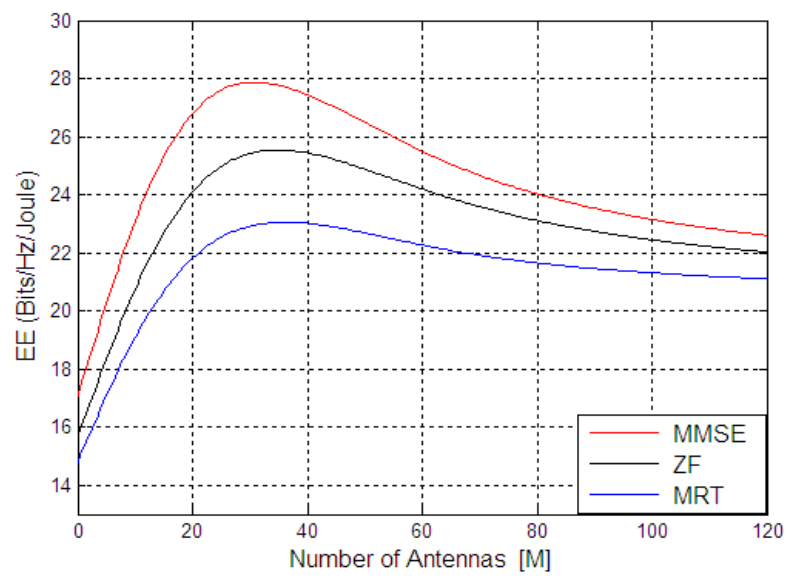

Figure 3. Energy efficiency with number of antennas elements $M$

\section{CONCLUSION}

Energy efficiency in a multiple cellular system is achieved by increasing the number of antennas at the BS. We have examined how to obtain maximum EE, which depends on selecting the optimal number of antennas and fixing the number of scheduled users inside cells. In addition, analyzing how to minimize total power consumption depends nonlinearly on $M$ and $K$. Total power consumption consists of circuit power from analog devices and power amplifiers in massive MIMO systems. The techniques of linear precoding beamforming, MMSEBF, ZFBF, and MRTBF enable minimum power transmission at the BS to select the best-performing active antenna, where EE is able to work at high SNR. Consequently, linear precoding MMSE achieves maximum EE, more so than ZF and MRT, because the MMSE is able to suppress 
interference actively and make a massive MIMO system less sensitive to SNR at an increased number of antennas.

\section{ACKNOWLEDGEMENTS}

The authors would like to thank the Universiti Tun Hussein Onn Malaysia for the generous financial support under the Contract Grant Scheme (U550).

\section{REFERENCES}

[1] G. Li, et al, "Energy-efficient wireless communications: tutorial, survey, and open issues," IEEE Wireless Communications, 2011, vol. 18, no.6, pp. 28-35.

[2] Z. Xu, et al, "Energy-efficient configuration of spatial and frequency resources in MIMO-OFDMA systems," IEEE Transactions on Communications, 2013, vol. 61, no. 2, pp. 564-575.

[3] E. Björnson, et al, "Designing multi- user MIMO for energy efficiency: When is massive MIMO the answer?," In Proc. IEEE. WCNC, 2014, pp. 242-247.

[4] S.K. Mohammed, et al, "Per-antenna constant envelope precoding for large multi-user MIMO systems," IEEE Transactions on Communications, 2013, vol. 61, no. 3, pp. 1059-1071.

[5] Y. Chen, et al, Characterizing energy efficiency and deployment efficiency relations for green architecture design," IEEE. International Conference on Communications Workshops. 2010, pp. 1-5.

[6] Y. Pei, et al, "How many RF chains are optimal for large-scale MIMO systems when circuit power is considered?," IEEE. In Global Communications Conference (GLOBECOM), 2012, pp. 3868-3873.

[7] H.Q. Ngo, et al, "Energy and spectral efficiency of very large multiuser MIMO systems," IEEE Transactions on Communications. 2013, vol. 61, no. 4, pp. 1436-1449.

[8] H. Yang, et al, "Total energy Efficiency of cellular large scale antenna system multiple access mobile networks, " In Proc. IEEE Online Green Comm, 2013, pp. 27-32.

[9] E. Björnson, et al, Optimal design of energy- efficient multi-user MIMO systems: Is massive MIMO the answer?," IEEE. Transactions on Wireless Communications.2015, vol. 14, no. 6, pp. 3059-3075.

[10] H.V. Cheng, et al, "Massive MIMO at night: On the operation of massive MIMO in low traffic scenarios," IEEE. International Conference on Communications (ICC). 2015, pp. 1697-1702.

[11] N. Jindal, "High SNR analysis of MIMO broadcast channels," in Proc.IEEE International Symposium on Information Theory (ISIT), pp. 2310- 2314, Adelaide, vol. 53, no. 12, 2005.

[12] A. M. Tulino, et al, "Random Matrix Theory and Wireless Communications," Now Publishers Inc., 2004.

[13] A. Pitarokoilis, et al, "Uplink performance of time- reversal MRC in massive MIMO systems subject to phase noise," IEEE. Transactions on Wireless Communications. 2015, vol. 14, no. 2, pp. 711-723.

[14] Y. Zhou, et al, "QoS-aware energy-efficient optimization for massive MIMO systems in 5G," in IEEE Wireless Communications and Signal Processing (WCSP), Oct.2014, pp. 1-5.

[15] Y.G. Lim, et al, "Performance analysis of massive MIMO for cell- boundary users, "IEEE Transactions on Wireless Communications, 2015, vol. 14, no. 12, pp. 6827-6842.

[16] T. Van Chien, et al, "Joint power allocation and user association optimization for massive MIMO systems," IEEE Transactions on Wireless Communications. 2016, vol. 15, no. 9, pp. 1-30.

[17] E. Björnson, et al, "Optimal multiuser transmit beamforming: A difficult problem with a simple solution structure [lecture notes]," IEEE Signal Processing Magazine. 2014, vol. 31, no. 4, pp.142- 148.

[18] L. Zhao, et al, "Energy efficient power allocation algorithm for downlink massive MIMO with MRT precoding," IEEE. In Vehicular Technology Conference (VTC Fall), 2013, pp. 1-5.

[19] T. Yoo, et al, "On the optimality of multi-antenna broadcast scheduling using zero-forcing beamforming," IEEE Journal on Selected Areas in Communications, 2006, vol. 24, no. 3, pp. 528-541.

[20] X.Gao, et al, "Linear pre-coding performance in measured very-large MIMO channels," IEEE. In Vehicular Technology Conference (VTC Fall). 2011, pp. 1-5.

\section{BIOGRAPHIES OF AUTHORS}

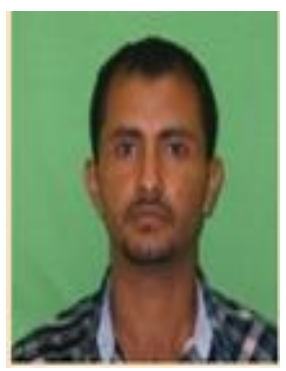

Adeeb Salh was born in Taiz, Yemen. He received his Bachelor of Electrical and Electronic Engineering from IBB University, Yemen in 2007. He then received his Master of Electrical and Electronic Engineering from Universiti Tun Hussein Onn Malaysia (UTHM), Malaysia in 2015. $\mathrm{He}$ is currently a PhD student at UTHM. His research works are related to the Massive MIMO systems. 

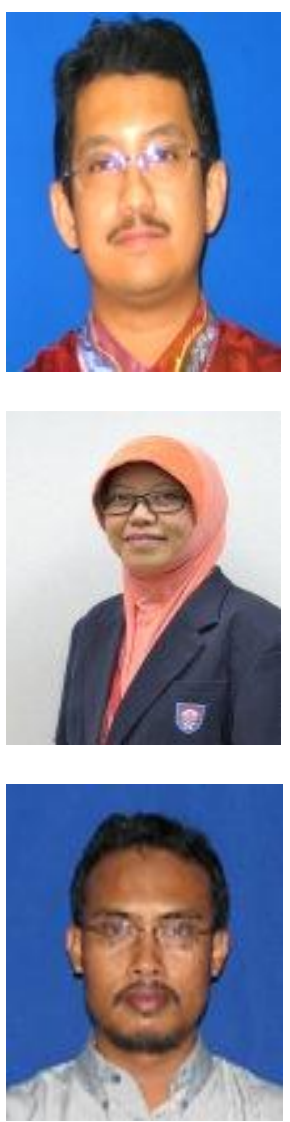

Lukman Audah was born in Kuala Lumpur, Malaysia. He received Bachelor of Engineering (Telecommunications) from Universiti Teknologi Malaysia in 2005. He then received his MSc. in Communication Networks and Software from University of Surrey, United Kingdom. He also received $\mathrm{PhD}$ in Electronic Engineering from University of Surrey. He is currently a lecturer in the Communication Engineering Department, Universiti Tun Hussein Onn Malaysia. His research interests are wireless and mobile communications, Internet traffic engineering, network system management, data security and satellite communications.

Nor Shahida Mohd Shah was born in Johor, Malaysia. She received Bachelor of Electrical and Electronics Engineering from Tokyo Institute of Technology, Japan in 2000. She then received MSc. in Physics from University of Malaya, Malaysia in 2003. She received PhD in Electrical, Electronics and System Engineering from Osaka University, Japan in 2012. She is currently a lecturer in the Communication Engineering Department, Universiti Tun Hussein Onn Malaysia. Her research interests are optical amplifiers and regenerators system design.

Shipun Anuar Hamzah was born in Parit Buntar, Malaysia. He received Bachelor of Electrical Engineering from Universiti Teknologi Malaysia in 1998. He then received MSc. in Communication and Computer from Universiti Kebangsaan Malaysia in 2000. He received PhD in Electrical Engineering from Universiti Teknologi Malaysia in 2014. His research interests are active antenna design, harmonic suppression antenna and energy harvesting. 